\title{
Oficinas Virtuales de Empleo, el Reto de Universalizar Los Servicios Publicos de Empleo (SPE)
}

\author{
José A. Climent Rodríguez* \\ Yolanda Navarro Abal***
}

\section{RESUMEN:}

El presente artículo se divide en dos partes, en primer lugar, y de manera introductoria, realiza una revisión del estado actual de implantación de la administración electrónica en nuestro país y por comunidades autónomas. En la segunda parte, se analiza en que medida los servicios públicos de empleo, tanto el estatal como el de las diversas comunidades autónomas han adaptado sus recursos, procedimientos y servicios al formato on line. En esta segunda parte, se realiza una comparativa las oficinas virtuales de empleo de los SPE españoles en cuanto a criterios de accesibilidad, tramitaciones on line o nivel de información, entre otros.

Palabras Clave:

Servicios públicos de empleo, administración electrónica, oficinas virtuales de empleo, empleo on line.

ABSTRACT:

This article is divided into two parts, first, and as an introduction, we review the current state of e-government implementation in our country and by region. In the second part, we analyze to what extent the public employment services, both state and that of the

* Profesor Asociado. Dpto. Psicología Clínica, Experimental y Social. U. de Huelva • jose.climent@dpsi.uhu.es

*: Profesor Contratado Doctor. Dpto. Psicología Clínica, Experimental y Social. Universidad de Huelva • yolanda.navarro@dpsi.uhu.es 
different regions have adapted their resources, processes and services online format. In this second part, a comparison is made virtual offices use of Spanish SPE in terms of accessibility criteria, procedures on line or level of information, among others.

KEYWORDS:

Public employment services, e-govermment, virtual office jobs, jobs on line.

\section{INTRODUCCIÓN: LA E-ADMINISTRACIÓN, UNA REALIDAD NADA VIRTUAL}

En apenas una década, y gracias al impulso de políticas europeas, la sociedad del conocimiento y de las tecnologías de la comunicación se ha ido materializado en diversas realidades que han significado, cuanto menos, la mejora en las condiciones de vida de los ciudadanos en relación al más eficiente acceso a la información y a los servicios. Uno de estos retos que parece haberse cumplido con creces es el referido a la implantación de la administración electrónica europea. Desde el año 2000, en el que apareció una primera iniciativa de envergadura desde el seno de la Comisión Europea intentando poner las bases de una economía basada en el conocimiento, pasando por los posteriores Planes de Acción eEurope, y hasta llegar al Plan de Acción de Administración Electrónica i2010, se han propuesto los pilares que han permitido, en la actualidad, poder hablar de una verdadera y, casi, generalizada utilización de servicios electrónicos públicos por parte de los ciudadanos europeos de la Europa de los 12, fundamentalmente. Precisamente, uno de los puntos sobre los que pone mayor énfasis este último plan de acción europeo, es la consecución de mayores cotas de eficiencia y eficacia por parte de los servicios públicos, gracias a un esfuerzo constante por utilizar la administración electrónica y reducir así las cargas administrativas, mejorando los procesos organizativos y promocionando una economía sostenible, baja en emisiones de carbono. La meta es lograr que, de aquí a 2015, el 50\% de los ciudadanos y el 80\% de las empresas utilicen los servicios de administración electrónica para trámites cotidianos. De esta forma, es posible que en un futuro no muy lejanos todo el abanico de servicios públicos se pueda realizar, y algunos de ellos exclusivamente, a través de internet, pasando antes por el reto de acercar el uso de la red de redes al conjunto de la población europea.

En este sentido, resulta notable el esfuerzo previsto para que los países miembros puedan desarrollar la oferta de servicios de administración electrónica en toda la Unión, y en particular, la carga añadida que soportan ciudadanos y empresas cuando se trasladan a otro país, con el obstáculo que esto supone para la competitividad económica europea. Así, de aquí y hasta el 2015 deberán surgir herramientas que permitan, por ejemplo, a los estudiantes matricularse por internet en cualquier universidad de la UE.

Por lo que a nuestro país se refiere, el documento e-Administración del Ministerio de Administraciones Públicas señala que la administración electrónica consiste en el 
uso de las tecnologías de la información y las comunicaciones en las Administraciones Públicas, para que, combinadas con ciertos cambios organizativos y nuevas capacidades de los empleados públicos, se incremente la eficacia, la productividad, la agilidad y la comodidad en la prestación de servicios a los ciudadanos. La entrada en vigor de la Ley 11/2007 de la administración electrónica (LAECSP), significa la concreción legal definitiva por la que el estado español hace patente su apuesta en el esfuerzo común europeo. Con la nueva Ley, se hace obligatorio proporcionar los servicios públicos por medios electrónicos y a través de ella, se establece, entre otras cuestiones; El soporte normativo y jurídico de la Administración Electrónica, los derechos de los ciudadanos, las obligaciones de las Administraciones Públicas, así como el fomento y uso de la interrelación a nivel tecnológico entre las diferentes Administraciones Públicas.

Se reconoce a los ciudadanos, a través de este referente normativo, el derecho a relacionarse con las Administraciones Públicas por medios electrónicos para ejercer los derechos reconocidos en la Ley de Régimen Jurídico y Procedimiento Administrativo Común, así como para obtener información, realizar consultas, alegaciones, solicitudes, consentimientos, pagos, etc. Además, se reconocen los derechos a elegir libremente el canal de acceso a los servicios, no aportar datos que ya obren en poder de las Administraciones Públicas, igualdad en el acceso, conocimiento del estado de tramitación de procedimientos, custodia por las administraciones de documentos electrónicos, identificación electrónica mediante DNI electrónico u otro certificado, seguridad y confidencialidad en el servicio, etc. En cuanto a las obligaciones de las Administraciones Públicas establece el deber de habilitar diferentes canales o medios que garanticen el acceso a los servicios electrónicos a todos los ciudadanos sin distinción. Dentro de dichos canales de acceso, se deben proporcionar al menos los siguientes: oficinas de atención presencial, atención personal y/o a través de medios electrónicos en la propia oficina para uso del ciudadano, puntos de acceso electrónico en forma de sedes electrónicas creadas y gestionadas por los departamentos y organismos públicos y disponibles para los ciudadanos a través de redes de comunicación. También prevé la creación de un Punto de acceso general para publicar la información sobre los servicios disponibles de toda la Administración General del Estado y sus Organismos Públicos, así como el acceso a los mismos desde dicho Punto (Ministerio de Política Territorial y Administración Pública, 2011).

En este contexto, hay que destacar una de las iniciativas recientes, auspiciadas por parte de la Dirección General para el Impulso de la Administración Electrónica (DGIAE) del Ministerio de Política Territorial y Administración Pública. Nos referimos al Portal de la Administración Electrónica (PAe,), que integra los contenidos y servicios de diversas fuentes públicas, centralizando en un único punto la información de las Administraciones Públicas en esta materia. Cabe destacar, dentro de este portal, el entorno CTT-PAe o directorio de iniciativas del CTT (Centro de Transferencia de Tecnología), que permite acceder a las diferentes iniciativas, proyectos y/o servicios, lo cual ayuda a la divulgación de las iniciativas en Administración Electrónica y a la reutilización de las mismas. 
Gráfico 1. Evolución del puesto de España en informes de la ONU

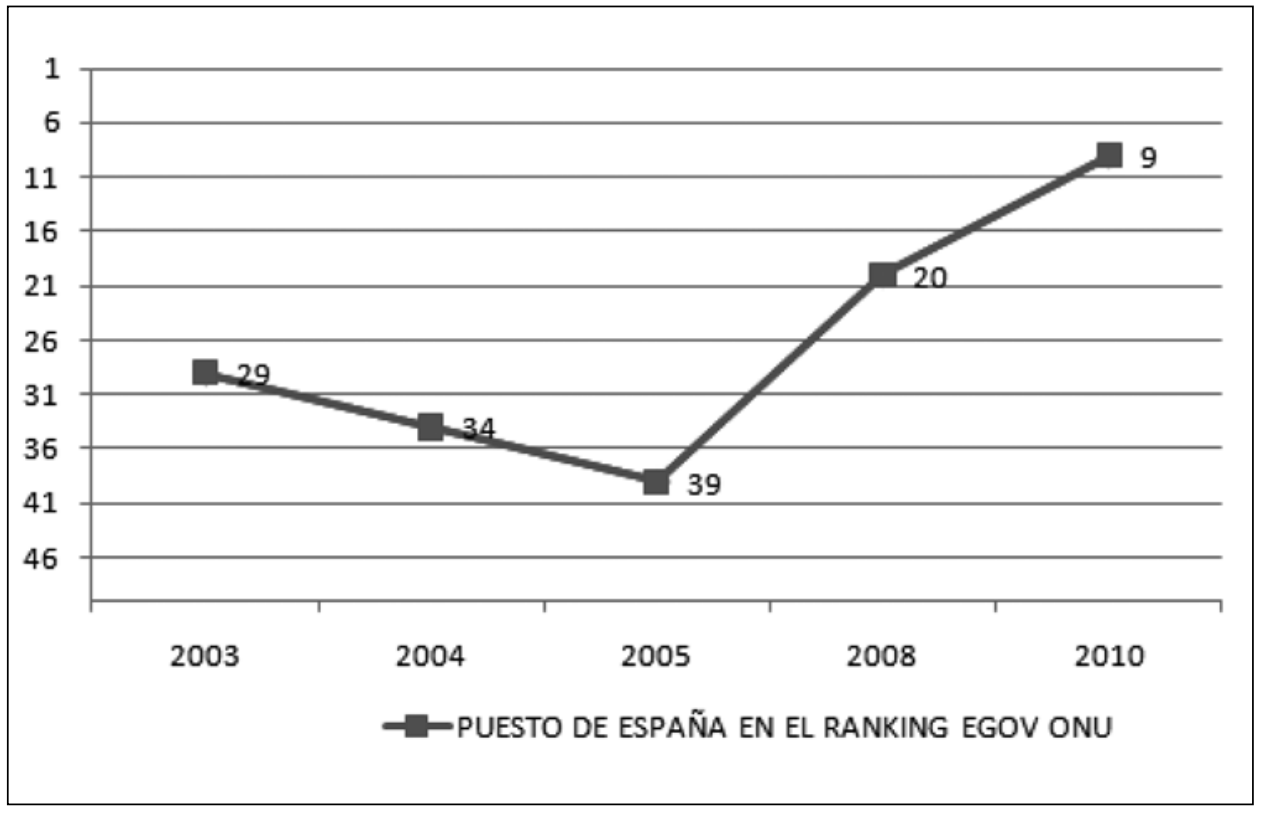

(Extraido del Resumen ejecutivo del Informe presentado al Consejo de Ministros de 16 de septiembre de 2011 sobre la situación de la Administración Electrónica en la Administración General del Estado)

Lo que resulta evidente, es que las Administraciones Públicas españolas han realizado un esfuerzo importante en los últimos años para garantizar a los ciudadanos su derecho a relacionarse electrónicamente con las Administraciones, un impulso que ha permitido alcanzar una posición de liderazgo en el desarrollo de la Administración Electrónica.

Los resultados de 2010 del informe "eReadiness" de la Organización de las Naciones Unidas (el más completo que se realiza a nivel internacional) sitúan a nuestro país en el noveno puesto del ranking mundial (el quinto en el subindicador específico de servicios electrónicos on-line) y en el quinto del europeo. España ha recibido un premio especial de la ONU por estos avances.

La Unión Europea (UE) también realiza desde hace una década un informe anual comparativo de Servicios Electrónicos sobre un conjunto de 20 servicios públicos (12 destinados a ciudadanos y 8 a empresas). Por primera vez, nuestro país se ha posicionado dentro de los 10 primeros puestos (concretamente en el octavo), tanto en disponibilidad como en sofisticación de servicios electrónicos. 


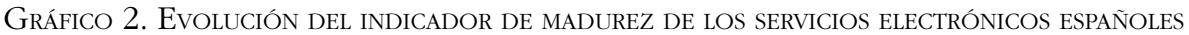
EN COMPARACIÓN CON LA MEDIA EUROPEA

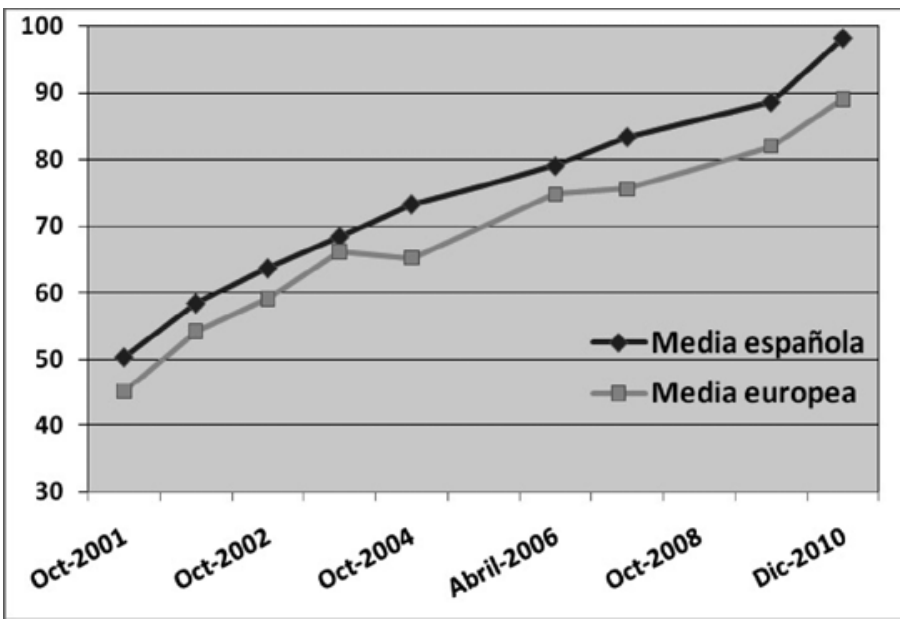

(Extraído del Resumen ejecutivo del Informe presentado al Consejo de Ministros de 16 de septiembre de 2011 sobre la situación de la Administración Electrónica en la Administración General del Estado)

En la actualidad, se han adaptado a la norma más de 2.300 procedimientos y servicios electrónicos en la AGE, lo que supone que, aproximadamente, un 90\% de sus procedimientos y un $99 \%$ de la tramitación total anual pueden realizarse ya por medios electrónicos. El cuadro adjunto permite apreciar el incremento en el número de procedimientos y servicios electrónicos para ciudadanos y empresas accesibles electrónicamente

\section{Gráfico 3. Evolución temporal de la adaptación de procedimientos en la AGE}

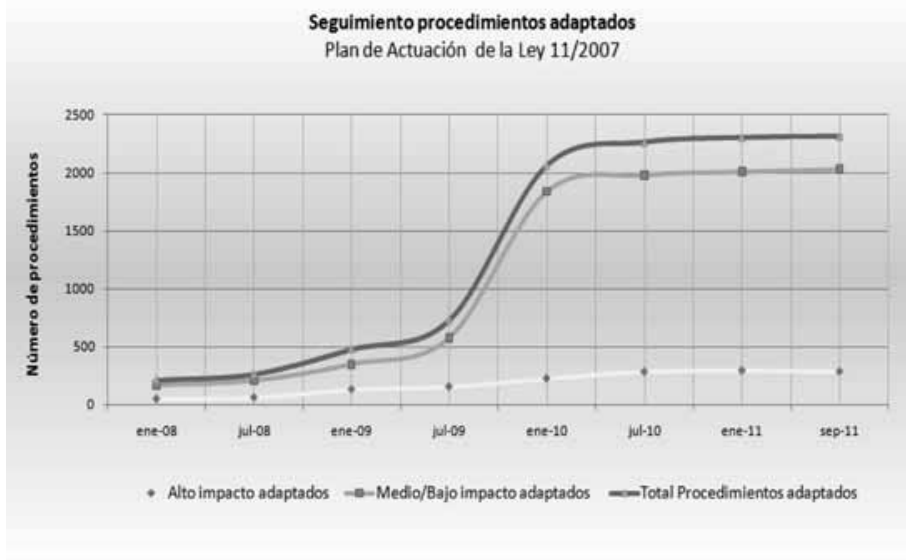

(Extraído del Resumen ejecutivo del Informe presentado al Consejo de Ministros de 16 de septiembre de 2011 sobre la situación de la Administración Electrónica en la Administración General del Estado) 
Por otro lado, destacar que España es líder mundial en el desarrollo de la firma electrónica. Las distintas autoridades de certificación existentes han expedido, a finales de 2010, más de dos millones de certificados electrónicos a personas físicas y 400.000 a entidades jurídicas. A finales de agosto de 2011 existen más de 24 millones de DNIe, Documentos Nacionales de Identidad Electrónicos, emitidos, esperando completar la expedición a toda la población en 2015.

El porcentaje de disponibilidad media online de los 26 servicios públicos considerados como más relevantes en las 17 Comunidades Autónomas y las 2 Ciudades Autónomas de Ceuta y Melilla era del 78\% en el primer trimestre del 2011. De ellos, los considerados servicios orientados a la ciudadanía alcanzaban el $81 \%$, mientras que los servicios orientados a empresas alcanzaban un $76 \%$ de disponibilidad online. Asturias y Madrid han alcanzado en este periodo la primera posición en el ranking con un 98\% de disponibilidad, siendo la brecha digital de 49 puntos entre ellas y Melilla, la última en el ranking. En general, los servicios públicos online ofrecidos a la ciudadanía están más desarrollados que los de empresas, si bien son 7 Comunidades autónomas las que alcanzan mayor porcentaje de disponibilidad online en servicios dirigidos a empresas que los dirigidos a ciudadanos (Asturias, Cantabria, Comunidad Valenciana, Galicia, Madrid, Murcia y Navarra). Ceuta y Melilla son las Comunidades que presentan el mayor diferencial entre el desarrollo de sus servicios a la ciudadanía y los dirigidos a empresas. Por último, cabe insistir en que, aún existen 9 Comunidades autónomas que están por debajo de la media global de disponibilidad (Capgemini Consulting, 2011).

\section{SERVICIOS PÚBLICOS DE EMPLEO EN INTERNET; DE LA INFORMACIÓN A LA TRAMITACIÓN}

Los Servicios Públicos de Empleo (SPE) desempeñan un papel importante en la ejecución de las políticas de empleo (Alujas, 2009). Tradicionalmente, estos servicios públicos han desarrollado su actividad en Europa en torno a tres grandes funciones:

- Como proveedor de información sobre el mercado de trabajo: Es decir, recogida de datos sobre ofertas de empleo y los candidatos potenciales y suministro de información sobre las posibilidades de formación o reconversión profesional;

- Como intermediador: Es decir, de dinamizador entre la oferta y la demanda de empleo en cada territorio.

- Como agente de ajuste del mercado laboral: los SPE, por estar involucrados en la implementación de las políticas de empleo, puede ayudar a ajustar la oferta y la demanda en el empleo.

Así, desde las distintas directivas europeas planteadas sobre empleo, en las dos últimas décadas, el papel esencial de los SPE se ha reflejado en cuatro áreas de acción prioritarias: - empleabilidad: los SPE desempeñan un papel clave en esta área, 
proporcionando asesoramiento sobre técnicas de búsqueda de empleo, el acceso a la formación profesional y la ayuda complementaria para grupos específicos; - el espíritu empresarial: la promoción de la auto-empleo para los desempleados o los trabajadores amenazados de despido es uno de los objetivos de la SPE; - capacidad de adaptación: a pesar de la magnitud del problema del desempleo significa que el enfoque SPE la mayor parte de su acción sobre los desempleados, que siguen desempeñando un papel insignificante lejos de la gestión del cambio estructural en las empresas (la participación de los SPE en los diferentes programas de formación, el reciclaje y la redistribución de los trabajadores que se ven obligadas a adaptarse a los cambios estructurales y están en peligro de perder sus puestos de trabajo); - la igualdad de oportunidades: los SPE están involucrados en la promoción de la participación femenina en el mercado laboral.

Una cuestión resulta común a toda la directiva surgida sobre Servicios Públicos de Empleo desde el año 1998, y es la importancia de la modernización y el fortalecimiento de los servicios para hacerlos eficaces frente a los rápidos cambios en el mercado laboral para los trabajadores y empleadores. Y esa adaptación a los nuevos desafíos y realidades sociales ha de venir, fundamentalmente, aunque no sólo, a través del desarrollo en el uso de las tecnologías de la información y la comunicación Además, dichas tecnologías facilitan la coordinación de las prestaciones por desempleo y las acciones de orientación y asesoramiento, sobre todo en los países donde el SPE no tiene una estructura integrada. Todos los Servicios Públicos de Empleo europeos están desarrollando sistemas de autoservicio para mejorar la atención a los usuarios. El elemento clave son las bases de datos sobre vacantes y demandantes de empleo. El principal medio para la puesta en marcha del autoservicio es Internet, variando el nivel de interactividad y las funciones disponibles según los países. Otros medios son los terminales de autoservicio y los call centres, proporcionando éstos información sobre los servicios ofrecidos por el SPE. El avance en el uso de los sistemas de autoservicio puede contribuir a la prevención de desajustes formativos, a descubrir cuellos de botella, a potenciar la movilidad ocupacional y geográfica y en definitiva a mejorar el funcionamiento de los mercados de trabajo y la cuota de mercado de los SPE (Gugerbauer, Natter y Haylon, 2001).

Dentro del Programa de modernización de los SPE incluido en el Programa Nacional de Reformas de España 2005 destaca la puesta en marcha del Sistema de Información de los Servicios Públicos de Empleo (SISPE). Dicho sistema debe permitir a los Servicios Públicos de Empleo estatal y autonómicos compartir una información básica y coordinada sobre políticas activas de empleo y prestaciones por desempleo. Los objetivos respecto a los trabajadores y a los empleadores son: promover la libre circulación y la movilidad laboral de los demandantes de empleo, favorecer la igualdad de oportunidades en el acceso al empleo e incrementar la capacidad de cobertura de puestos de trabajo. Por otra parte, los objetivos respecto al Sistema Nacional de Empleo son: compartir la información y mejorar la capacidad de intermediación laboral, coordinar la gestión de los 
SPE, respetar diversidad territorial y la autonomía de gestión y asegurar la elaboración de estadísticas nacionales sobre el funcionamiento del mercado de trabajo. Por tanto, la implantación del SISPE pretende mejorar el servicio al ciudadano, facilitar la modernización de los SPE, disponer de información integrada y homogénea, mejorar la gestión de los programas comprometidos en el Programa Nacional de Reformas y garantizar la justificación puntual de acciones cofinanciadas por Fondo Social Europeo. Los avances en la implantación de dicho sistema se concretan en la introducción del registro informático en la totalidad de la contratación en España, la posibilidad de acceder a través de internet a las ofertas y demandas de empleo y a las oportunidades de formación en todo el territorio nacional, así como la creación de un portal de empleo que permita el contacto directo entre trabajadores y empresarios (permitiendo a los trabajadores introducir sus currículums, acceder a ofertas y contactar con los empleadores, y que estos incluyan sus ofertas, seleccionen candidatos y contacten con ellos).

\section{Gráfico 4. Mapa de Servicios Públicos de las Comunidades Autónomas}

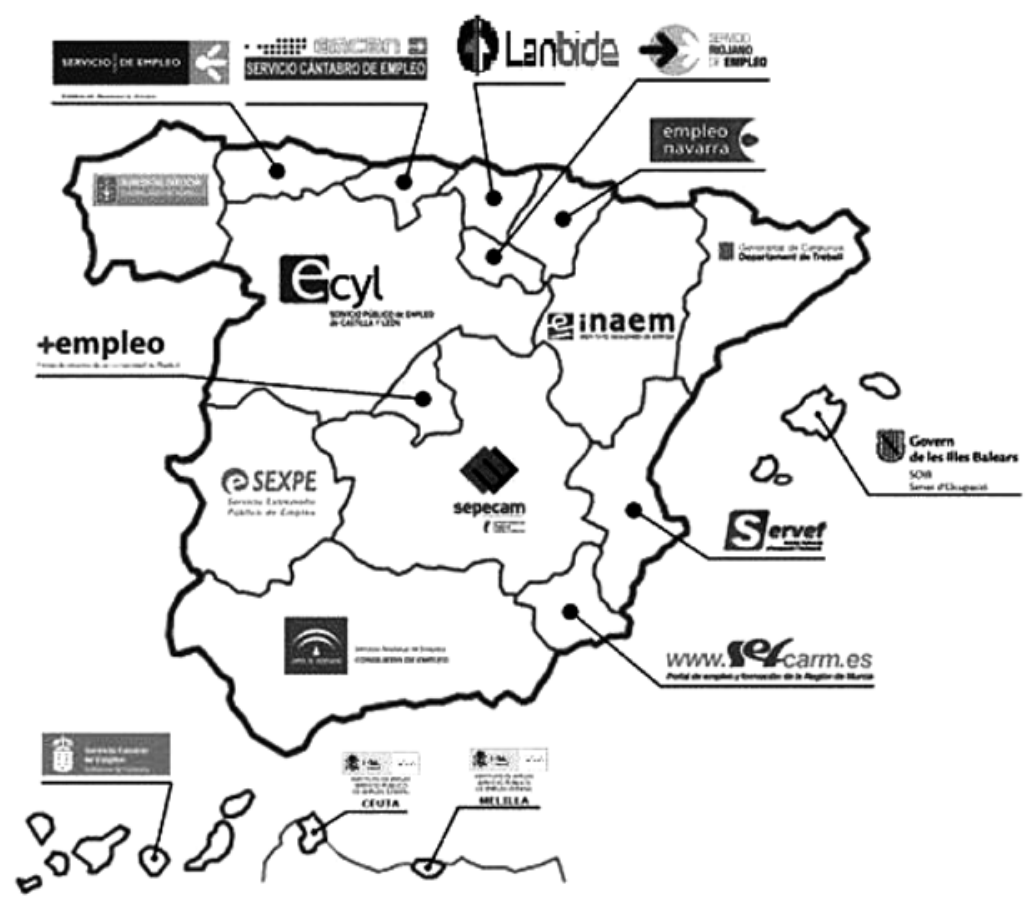

(Extraído: Elaboración propia) 
En la actualidad, la práctica totalidad de los Servicios Públicos de Empleo existentes en España, tienen un sitio web desde el que acercar, desde internet, sus servicios a la ciudadanía. En este sentido si es necesario realizar una diferenciación entre aquellos SPE que plantean su estrategia de posicionamiento en internet con un objetivo más cercano a la información de recursos, servicios, actividades y procedimientos, y aquellos otros que han establecido el proceso de implantación en la red como una oportunidad para, además de la información, que se establezca un amplio abanico de servicios y e-tramitaciones, aprovechando el potencial de las TICs para mejorar la prestación de servicios a ciudadanos y empresas, y para incorporar ciertos elementos tecnológicos como ventaja competitiva para los usuarios. Esta última visión de los servicios de empleo en internet, es lo más parecido a una Oficina Virtual de Empleo.

Durante el primer semestre del año 2011, los autores de este artículo realizaron una revisión de las 17 páginas de internet de los Servicios Públicos de Empleo Autonómicos, además de la página del Servicios Público de Empleo Estatal (SEPE). Se establecieron diversos criterios que permitieran establecer qué grado de bondad tenía cada uno de los servicios virtuales de empleo, entendida esta como; servicios disponibles, grado en que se puede interrelacionar con estos servicios, posibilidad de tramitaciones a través de la página y accesibilidad a la información.

En cuanto a los servicios disponibles, cabe destacar que, en todos los casos (18), aparece información denominada de localización de servicios. Es decir, directorios de localización de las oficinas y centros físicos del servicio de empleo en cuestión de entidades colaboradoras. De la misma forma, las 18 páginas virtuales de empleo establecen un combo de información general acerca del propio Servicio Público de Empleo, a modo de presentación institucional, aunque no en todos los casos esta información viene precedida de información sobre cómo navegar en la página, o el directorio de servicios ofrecidos por el propio Servicio Público de Empleo y los ofrecidos desde la página web en cuestión.

En cuanto a información sobre ofertas de empleo, los 18 e-SPE ofrecen información a sus ciudadanos sobre ofertas de empleo en el territorio, aunque durante el periodo consultado, sólo una Oficina Virtual de Empleo, la del Servicio Andaluz de Empleo, permitía al usuario inscribirse a través de la página en las ofertas.

En todos los casos analizados, las páginas webs permitían obtener información acerca de cómo buscar empleo, aunque la calidad, cantidad y accesibilidad de este tipo de información varía enormemente entre unas y otras. En algún caso se plantea como un link específico dentro de la página que se denomina literalmente así "buscar empleo" (el caso del Servicios Andaluz de Empleo) o a través de denominaciones sinónimas relacionadas con los pasos que el usuario puede dar en la página para mejorar la empleabilidad (como el caso de trabajastur, que lo denomina "déjate 
guiar"). En el resto de páginas de e-SPE aparecen enlaces a recursos relacionados con la búsqueda de empleo, pero sin un "hilo conductor" que los relacione de manera diacrónica. En general, todos estos recursos de orientación, formación, ofertas de empleo, etc. los engloban en un macro-apartado denominado "ciudadanos", en el que el usuario podrá encontrar recursos de interés para su inserción y/o cualificación.

Otro gran bloque de recursos en los e-SPE es el dirigido a informar a empresas acerca de los pasos para buscar trabajadores, incentivos a la contratación, legislación laboral o comunicación de contratos. Del mismo modo que en el apartado de información a ciudadanos, en el caso de las empresas, en general, las webs se limitan a ofrecer información en estas materias arriba descritas, y en sólo 6 de las 18 webs analizadas puede la empresa, además, realizar alguna tramitación relativa (comunicación de la contratación temporal, realización de trámites para solicitar incentivos a la contratación, solicitud de formación, etc...).

Según los casos, en estos apartados de información a empresas suele aparecer información específica para trabajadores autónomos (como darse de alta, legislación específica, información sobre tramitaciones e incentivos, formación para el colectivo, etc). En el período analizado, el 58\% de las webs de empleo tenían en el mismo apartado información, de manera indistinta, a empresas y autónomos, estando en el resto de las webs recogidos como apartados de información diferenciados.

En todas las páginas analizadas aparecen apartados específicos en el que se citan diversas informaciones de interés para los usuarios y que tienen un carácter muy temporal. Pueden aparecer como "noticias, eventos, tablón, novedades, etc...". El tipo de información contenida en estos apartados es, en general, muy diversa, aunque algunas páginas tienen tendencia a plantear noticias más de tipo institucional, otras páginas aprovechan este link para destacar datos de empleo y de la gestión del mismo de esa comunidad autónoma, ofertas de empleo, cursos de formación vigentes, programas y convocatorias de empleo, e incluso noticias relacionadas con procesos de reclutamiento y selección privados o de otras comunidades autónomas. En general, de los 18 sitios de novedades analizados durante el primer semestre de 2011, todos los contenidos mostrados se encontraban actualizados y vigentes. La cosa varía al realizar una búsqueda de recursos en los sitios específicos de cada página. En esos casos, suelen seguir publicados recursos que se encuentran desactualizados, especialmente recursos formativos fuera de plazo y, en menor medida, ofertas de empleo que aunque no tienen fecha de caducidad, superan en algunos casos más de tres meses desde su publicación.

Una de las características diferenciales entre webs de SPE con un objetivo eminentemente informativo, y aquellas otras que tienen una clara vocación de oficina virtual de servicios, quizás tenga que ver con la cantidad y calidad de tramitaciones 
que el usuario pueda realizar a través de ella. Así mismo, probablemente el uso de interfaces específicos para trabajar a través de la propia web también hablará del nivel de conectividad que permite. En junio de 2011, todas las páginas web de Servicios Públicos de Empleo analizadas permitieron realizar las siguientes gestiones al usuario/ demandante de empleo:

- Alta, Baja o Suspensión de su Demanda

- Renovación de Demanda

- Consulta de Demanda

- Duplicado de Tarjeta de Demanda

- Modificación de Datos Personales

- $\quad$ Solicitud de servicios.

- Informes de Demanda Personalizados

- Informe de periodo ininterrumpido inscrito en situación de desemple

- Informe de ocupaciones demandadas

- Informe de servicios y especialidades formativas requeridas

- Informe de periodos de inscripción

Previamente a la realización de estas e-gestiones, la persona usuaria debe registrarse, o a través de su identidad electrónica o aportando unas claves y contraseñas que previamente ha debido solicitar, para acceder a una interface en la que realizar las tramitaciones. En algunas webs, esta interface es la que denominan como oficina virtual electrónica. Probablemente esta acepción no sea del todo adecuada, ya que la mayoría de las páginas webs de Servicios Públicos de Empleo analizadas contienen otros interfaces o links de tramitación, con lo cual, toda la web y su entorno sería considerado oficina virtual.

Además, más del 60\% de las webs cuenta con herramientas tutorizadas para que la persona usuaria pueda realizar un currículum con formato europeo, dando opciones de guardar, modificar, y actualizar el currículum. De ellas, tan sólo cuatro permiten al demandante utilizar el currículum elaborado para realizar candidaturas directas a ofertas de empleo publicadas en la propia web.

Las mismas webs que ofrecen la posibilidad de tramitar candidaturas a ofertas de empleo, es decir 4, permiten además que los usuarios puedan realizar tramitaciones relacionadas con la preinscripción o inscripción directa a cursos de formación para el empleo. Excepción hecha de los programas de formación en alternancia, Escuelas Taller, Talleres de Empleo y Casas de Oficios, que en ninguna de las webs es posible una preinscripción, y menos aún, la inscripción electrónica. 
En cuanto a las tramitaciones que las empresas pueden realizar en las páginas webs de los SPE, cabe destacar que, en todas ellas se habilitan enlaces al programa Contrat@, en la web del Servicio Público de Empleo Estatal (SEPE). Se trata de un servicio web que permite a los empresarios que actúan en nombre propio, empresas y profesionales colegiados que actúen en representación de terceros, comunicar el contenido de la contratación laboral a los Servicios Públicos de Empleo desde su propio despacho o sede profesional. La utilización de este servicio requiere disponer de una Autorización de los Servicios Públicos de Empleo. Además, algunos SPE tienen un programa de gestión de contratos propio, al que se puede acceder de igual modo a través de su web.

Tal y como se refleja en el informe de fundación Orange y Capgemini Consulting, en el que se analiza el grado de desarrollo de servicios públicos online alcanzado por las comunidades autónomas españolas hasta el primer semestre de 2011, la oferta de servicios electrónicos por parte de estas administraciones públicas se ha incrementado y desarrollado de forma notable en las últimos años, y responsables en gran medida de esta realidad son los cambios introducidos en el "back-office" de las propias administraciones, donde se están gestionando cambios organizativos profundos y adaptaciones muy significativas hacia formas de trabajo con un alto componente tecnológico, y todo ello en consonancia con las directrices europeas en la materia. Este reto, asumido sin ambages por la casi totalidad de las administraciones públicas, que supone la puesta a disposición de empresas y ciudadanía de infraestructuras y sistemas de información optimizados en su funcionamiento, para proporcionar servicios de alta calidad y valor añadido, tiene que acompañarse, necesariamente de la necesaria cualificación de los empleados públicos. El personal de la Administración Pública es fundamental para la consecución de una e-administración universal y de calidad. El caso de los e-SPE en nuestro país, como ya hemos comprobado a lo largo del artículo, sigue una evolución muy pareja al del resto de la administración electrónica. La comunidad autónoma que ha apostado claramente por la innovación en su administración online, destaca en cuanto a la prestación y tramitación a través de internet en la mayor parte de sus servicios públicos, y entre ellos, claro está, también en los de empleo. No resulta casual que las comunidades autónomas que ocupan las primeras posiciones en el ranking de servicios públicos online durante 2010 y primer trimestre de 2011, que son Asturias, Madrid y Andalucía, presenten también unos e-SPE más interactivos, con mayor número de tramitaciones online y que algunas de ellas, como el caso del Servicio Andaluz de Empleo, se esté trabajando desde hace tiempo en la línea de incorporar estrategias de web 2.0 a las formas de gestionar el empleo desde internet, posicionándose en twitter, facebook, linkedin, canal en youtube, blogs colaborativos y, en general buscando nuevas fórmulas de relación más lineales entre administración pública y ciudadanía (Montaner, 2010). 


\section{BIBLIOGRAFÍA}

Alojas Ruiz, J.A. (2009): "Servicios públicos de empleo e intermediación laboral en la Unión Europea”, Ekonomiaz, n 71.

Capgemini Consulting: Estudio Comparativo 2011 de los Servicios Públicos online en las Comunidades Autónomas Resultados $5^{a}$ Medición. Abril de 2011. Disponible en: http://www.informeeespana.es/docs/Estudio Comparativo 2011 Servicios on line.pdf

Gugerbauer, I., Natter, M. y Naylon, I. (2001): Self-Service Systems in European Public Employment Services, Viena, ÖSB.

Ministerio de Política Territorial y Administración Pública. Secretaría de Estado para la Función Pública. Gobierno de España (2011): Resumen Ejecutivo del informe presentado al Consejo de Ministros de 16 de septiembre de 2011 sobre la situación de la administración electrónica en la administración general del estado. Disponible en: http://administracionelectronica.gob.es/? nfpb=true\& pageLabel=P603 $\underline{65901274269230261 \& l a n g P a e=e s}$

Montaner, J. (2010): "Introduciendo Herramientas 2.0 en los Servicios Públicos de Empleo en Andalucía". Revista Signos Universitarios Virtual. no 9. Disponible en:http://www.salvador.edu.ar/vrid/publicaciones/Montaner--.pdf 